\section{Allergologisches Nachschlagewerk für die Praxis}

\begin{abstract}
- If Jahre nach Erscheinen der Erstauf- lage liegt die „Praktische Allergologie“ nun in gründlich überarbeiteter Form vor. Zwischenzeitlich haben allergische Erkrankungen quantitativ einen noch höheren Stellenwert erhalten, das Spektrum diagnostischer und therapeutischer Möglichkeiten hat sich ebenfalls erweitert. Aufgrund der Vielzahl von Manifestationsformen der Allergie sind sehr unterschiedliche medizinische Fachbereiche involviert, dabei den interdisziplinären Überblick zu behalten ist für den „Praktiker“ kaum umsetzbar. Hier setzt das
\end{abstract}

vorliegende Buch sehr praxisnah an. Die einzelnen Beiträge dieses Multiautorenwerks decken das gesamte Spektrum möglicher Symptome und Krankheitsbilder vom Abdominalschmerz beim LatexNahrungsmittel-Allergiesyndrom bis zur Zimmerspringbrunnenalveolitis ab. Neben Genetik und Umwelteinflüssen werden die immunologischen Grundlagen, die allergischen Krankheitsbilder nach Fachgebieten bzw. Organen geordnet, Diagnostik und Therapie kurz und gut verständlich dargestellt. Jedes Hauptkapitel wird durch ein übersichtliches und

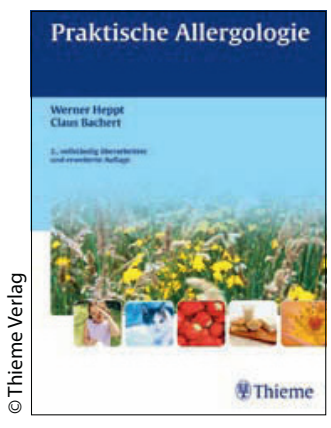

W. Heppt und C. Bachert (Hrsg) Praktische Allergologie 2. Auflage Thieme Verlag, 2011; 448 Seiten; 129,95 Euro ISBN 978-3-13106812-5

weitgehend aktuelles Literaturverzeichnis ergänzt. Als aktuelles Nachschlagewerk kann das Buch dem allergologisch tätigen Arzt vorbehaltslos empfohlen werden.

\title{
Schwierige Gespräche meistern
}

K ommunikation mit „schwierigen“ (und nicht-schwierigen Patienten) ist ein wichtiges Instrument in einer Arztpraxis oder im Krankenhaus. Wer die Grundlagen der Kommunikation kennt und anwendet und dabei sein Gegenüber besser einschätzen kann, trägt wesentlich dazu bei, eine Gesprächssituation positiv $\mathrm{zu}$ beeinflussen und wird in schwierigen Kontakten mehrere Lösungswege kennen und anwenden können.

Wichtig ist immer, der Ursache für eine gestörte Kommunikation auf die Spur zu kommen. Oft sind die Patienten gar nicht schwierig, sondern werden es nur durch ungeeigneten Umgang, mangelnde Achtsamkeit und schlechtes Zeitmanagement.

Die Autoren, Joachim Sandner und Volker Faust, vermitteln einen Überblick über die Grundlagen der Kommunikation speziell ausgerichtet auf den Patientenkontakt und eine umfassende Darstellung relevanter Störungsbilder aus dem seelischen und psychosozialen Bereich. Das Buch richtet sich an verschiedene Zielgruppen: Fachärzte, Arzthelferinnen, Klinikärzte, Schwestern und Pfleger, Mit-

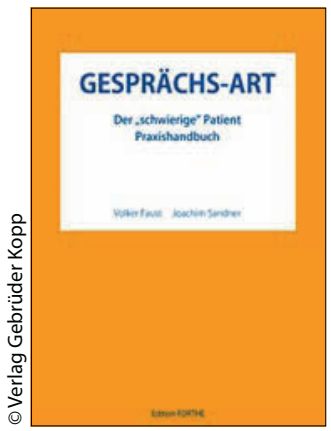

V. Faust, J. Sandner Gesprächs-Art, Der "schwierige" Patient Verlag Gebrüder Kopp, 2010 700 Seiten 49,90 Euro ISBN 978-3926509-33-8

arbeiter von Pflegeeinrichtungen. Mit seinem ausführlichen Sachwortverzeichnis dient es auch als Nachschlagewerk im Praxis- und Klinikalltag.

\section{gz}

\section{Einsatzmöglichkeiten der Neuraltherapie}

D as Buch gibt dem Leser aus unterschiedlichen Perspektiven umfassende Einblicke in die Einsatzmöglichkeiten der Neuraltherapie. Beginnend mit einem Überblick über die historische Entwicklung und den Stellenwert der Neuraltherapie in der Geschichte der Medizin vermitteln die folgenden Kapitel detailliert die für das Verständnis der Neuraltherapie wesentlichen Grundlagen aus Anatomie, Physiologie und Pharmakologie.

Nach einem weiteren, sehr anschaulichen Kapitel über die diagnostischen Verfahren wird der Leser im Kapitel „Injektionstechniken“ durch die neuraltherapeutisch zugängliche Anatomie "geleitet" und zwar unter Angabe von Indikationen, Kontraindikationen und spezieller Wirkungsweise.

„Neuraltherapie in den Fachgebieten“ stellt dann noch einmal den unmittelbaren Bezug zu den einzelnen Fachgebieten her und listet die jeweils möglichen und vor allem sinnvollen Indikationen auf. Abschließend findet der Leser die Indikationen alphabetisch geordnet, unter Angabe von Behandlungsplanung und begleitenden Therapieoptionen. Für die Anwendung des Buchs in der Praxis ergibt sich daraus die Problematik, dass sich der Leser die benötigten Informationen zu einem Krankheitsbild immer aus unterschied-

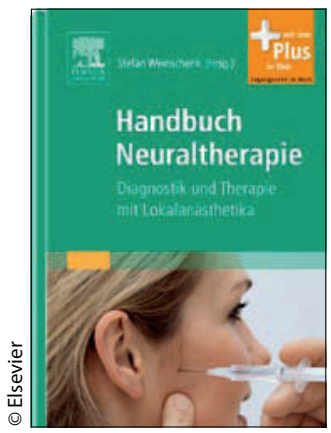

S. Weinschenk (Hrsg.) Handbuch Neuraltherapie Elsevier 2010 1.120 Seiten 129,00 Euro ISBN 978-3-43758210-3

lichen Kapiteln zusammensuchen muss. Insgesamt ist das Buch als Nachschlagewerk vor allem für die Kolleginnen und Kollegen unverzichtbar, die ihren Patienten die Neuraltherapie als Therapieoption anbieten möchten. 